\title{
Black Cohosh (Actaea racemosa L.) Improves Serum Lipid Profiles and Vasomotor Responses in Ovariectomized Rats
}

\author{
Eun-Young Kim ${ }^{1}$, Ah-Young Song ${ }^{1}$, Yiseul Kim ${ }^{1}$, Byung-Koo Yoon ${ }^{2}$, YoungJoo Lee ${ }^{3}$, Mee-Ra Rhyu ${ }^{1, *}$ \\ ${ }^{1}$ Division of Functional Food Research, Korea Food Research Institute, Gyeonggi-do, Republic of Korea \\ ${ }^{2}$ Samsung Medical Center, Sungkyunkwan University School of Medicine, Seoul, Republic of Korea \\ ${ }^{3}$ Department of Bioscience and Biotechnology, College of Life Science, Sejong University, Seoul, Republic of Korea \\ *Corresponding author: mrrhyu@kfri.re.kr
}

\begin{abstract}
We investigated the effects of long-term administration of black cohosh extract (BcEx) on serum lipid profiles and vasomotor responses in ovariectomized (OVX) rats and compared them with those of rats administered $17 \beta$-estradiol (E2) or raloxifene, a selective estrogen receptor modulator. Vehicle (OVX- or sham-control), BcEx $(0.5$ or $3.0 \mathrm{mg} / \mathrm{kg} / \mathrm{day}), \mathrm{E} 2(0.5 \mathrm{mg} / \mathrm{kg} / \mathrm{day})$, or raloxifene $(2.5 \mathrm{mg} / \mathrm{kg} / \mathrm{day})$ were injected subcutaneously for 5 weeks, and serum lipid profiles and vasomotor responses were measured at the end of the treatment. BcEx lowered total cholesterol (TC) and low-density lipoprotein cholesterol (LDL-C) levels, but did not affect high-density lipoprotein cholesterol or triglyceride (TG) levels. Raloxifene showed a similar effect to that of BcEx, while E2 attenuated the increase in TC and LDL-C levels and significantly increased TG levels. The vascular relaxation induced by carbachol increased significantly in norepinephrine-precontracted aortic rings isolated from BcEx-, E2-, or raloxifene-treated rats. No change in uterine weight was observed in the BcEx-treated group. The raloxifene-treated group showed a similar trend as that of the BcEx-treated group, but E2 significantly increased uterine weight. These results suggest that long-term administration of BcEx behaves similar to the selective estrogen receptor modulator raloxifene.
\end{abstract}

Keywords: black cohosh, ovariectomy, lipid profiles, vasomotor responses, selective estrogen receptor modulators

Cite This Article: Eun-Young Kim, Ah-Young Song, Yiseul Kim, Byung-Koo Yoon, YoungJoo Lee, and Mee-Ra Rhyu, "Black Cohosh (Actaea racemosa L.) Improves Serum Lipid Profiles and Vasomotor Responses in Ovariectomized Rats." Journal of Food and Nutrition Research, vol. 5, no. 8 (2017): 539-544. doi: $10.12691 /$ jfnr-5-8-2.

\section{Introduction}

Estrogen deficiency after menopause causes numerous changes in estrogen-receptive tissues, such as the brain, bones, and the cardiovascular system [1]. Estrogens are believed to be important in preventing cardiovascular diseases [2]; therefore, hormone replacement therapy (HRT) has been used to alleviate postmenopausal symptoms and prevent associated diseases [3]. Because of the controversy over the adverse effects and risks of HRT, alternative and complementary medicines are now used commonly. Selective estrogen receptor modulators (SERMs), which function as either estrogen receptor agonists or antagonists, have become popular as alternatives to HRT [4]. SERMs have beneficial estrogenic effects on the cardiovascular system [5]. Cardioprotective effects of SERMs, including hypolipidemic and vasorelaxant effects, have also been reported [6,7].

The rhizomes of Actaea racemosa L. or Cimicifuga racemosa (L.) Nutt. (Ranunculaceae), commonly known as black cohosh, have been used conventionally as a remedy for menstrual cramps, pain during parturition, and climacteric complaints, including hot flashes, irritability, mood swings, and sleeping disorders [8]. A number of clinical trials have examined the efficacy and safety of black cohosh $[9,10]$ and reported estrogenic effects in bones and fat [11], as well as attenuated body weight gain and intra-abdominal fat accumulation in ovariectomized (OVX) rats [12]. However, the mechanism underlying the effectiveness of black cohosh in treating menopausal symptoms is unclear, and its effects remain controversial. We reported previously that extracts of black cohosh (BcEx) elicit endothelium-dependent vasorelaxation in rat aorta by enhancing activity of the nitric oxide (NO)/cGMP system [13]. NO is a key regulator of endothelial function and mediator of the vascular benefits of estrogen [14]. Moreover, raloxifene (RLX), a second-generation SERM, activates endothelial NO synthase [15]. This study investigated the effects of BcEx on serum lipid profiles and vasomotor responses to carbachol in isolated aortic rings from OVX rats precontracted with norepinephrine (NE). We compared the effects to those of $17 \beta$-estradiol (E2) and RLX. 


\section{Materials and Methods}

\subsection{Reagents}

Carbachol, DMSO, E2, NE, PEG 400, and RLX were purchased from Sigma Chemical Co. (St. Louis, MO, USA). Ketamine (Yuhan Co., Seoul, Korea) and xylazine (Bayer Korea Ltd., Gyeonggi-do, Korea) were kindly supplied by Prof. Dr. Bo-Kyung Kim, Department of Physiology, School of Medicine, Konkuk University (Seoul, Korea).

\subsection{Plant Extract}

The powdered BcEx used in this study was a standard one utilized as the raw material of a dietary supplement. The sample was supplied from the NIH Center for Botanical Dietary Supplements Research at the University of Illinois at Chicago (UIC) and prepared and characterized as previously described $[13,16]$. Briefly, black cohosh rhizomes/roots were provided by Pure World Botanicals, Inc. (South Hackensack, NJ; lot 9-1744) [17], and were botanically verified by the UIC/NIH Center for Botanical Dietary Supplements Research and characterized by PCR [18]. A voucher specimen (BC001) has been deposited at the Program for Collaborative Research in the Pharmaceutical Sciences (PCRPS), University of Illinois at Chicago. Milled roots/rhizomes of black cohosh was extracted by percolation with $75 \%$ ethanol and dried in vacuo. BcEx was stored in a desiccator at room temperature and dissolved in $1.25 \%$ DMSO in PEG 400.

\subsection{Animal Treatment and Aortic Rings Preparation}

Twelve-week-old female Sprague-Dawley (SD) rats (260 to $280 \mathrm{~g}$ ) were obtained from Hanlim Laboratory Animal Co. (Gyeonggi-do, Korea). Animals were fed a standard laboratory rat chow (PICO-LAB Rodent Diet 205053, PMI Feeds, Richmond, IN, USA) for a 24 h, 1-week period of acclimatization with tap water. Diet and water were administered ad libitum. The air-conditioned animal room was maintained at $22 \pm 2{ }^{\circ} \mathrm{C}$, with a relative humidity

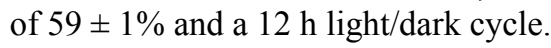

One week after acclimatization, $48 \mathrm{SD}$ rats were randomly assigned to one of the six groups. Five groups were surgically ovariectomized, and one group was sham-operated under anesthesia using ketamine $(70 \mathrm{mg} / \mathrm{kg})$ and xylazine $(10 \mathrm{mg} / \mathrm{kg})$ and allowed to recover for 2 weeks. The OVX SD rats were randomly assigned to one of the five treatment groups. The first group received subcutaneous injections of the vehicle (OVX-control; 100 $\mu \mathrm{L}$ of $1.25 \%$ DMSO in PEG 400) daily; the second group received daily injections of E2 $(0.5 \mathrm{mg} / \mathrm{kg} /$ day in $100 \mu \mathrm{L}$ of a vehicle); the third group received RLX $(2.5 \mathrm{mg} / \mathrm{kg} /$ day in $100 \mu \mathrm{L}$ of a vehicle); the fourth and fifth groups received $\operatorname{BcEx}(0.5$ and $3.0 \mathrm{mg} / \mathrm{kg} /$ day in $100 \mu \mathrm{L}$ of a vehicle, respectively) for 5 weeks. Sham-operated rats (shamcontrol) received vehicle alone. The body weight of the $\mathrm{SD}$ rats was recorded once a week. At the end of the treatment, bloods were taken, rats were sacrificed, and tissue samples were obtained after overnight fasting. The care and use of the animals followed out institutional and national guidelines, and the protocol was approved by the committee on the Ethics of Animal Experiments of the Korea Food Research Institute (Permit Number: KFRI-M12028).

\subsection{Measurement of Serum Lipid Profiles}

For the measurement of serum lipid profiles, bloods were drawn from the orbital sinus vein of rats. The blood samples were centrifuged at 3,000 rpm for $10 \mathrm{~min}$ to collect the serum, and were stored at $-20^{\circ} \mathrm{C}$ until further study. Uteri were dissected free of fat, and wet weights were measured. Total cholesterol (TC), triglyceride (TG), and high-density lipoprotein cholesterol (HDL-C) levels in the serum were determined using automatic dry chemistry analyzer for veterinary (Fuji Photo Film Co., Japan). The concentration of low-density lipoprotein cholesterol (LDL-C) was calculated using the formula of Freidwald et al. [19].

\subsection{Vasomotor Responses to Carbachol in NE-precontracted Isolated Aortic Rings}

The thoracic segment of aorta was dissected from the surrounding connective tissues and cut into rings $2-3 \mathrm{~mm}$ in length. The rings were then transferred to $3-\mathrm{mL}$ horizontal-type muscle chambers, and bathed in physiological salt solution (PSS) containing (mM) $\mathrm{NaCl}$ (115), $\mathrm{KCl}$ (5), $\mathrm{CaCl}_{2}$ (2.1), $\mathrm{MgSO}_{4}$ (1.2), $\mathrm{NaHCO}_{3}$ (25), glucose (11), and $\mathrm{KH}_{2} \mathrm{PO}_{4}(1.2)$ at $37^{\circ} \mathrm{C}$, in an atmosphere of $95 \% \mathrm{O}_{2}$ and $5 \% \mathrm{CO}_{2}$. The rings were mounted on stainless steel hooks, connected to a force-displacement transducer (FT03; Grass, West Warwick, RI, USA), which was connected to a polygraph system (RPS 212; Grass) and a computer analyzer (Power Laboratory 400, MacLab; AD Instruments, Castle Hill, Australia). A basal tension of $1 \mathrm{~g}$ was applied and each experiment was performed on rings prepared from different rats.

All rings were equilibrated for 60 min under a resting tension of $1 \mathrm{~g}$ and then $72 \mathrm{mM} \mathrm{KCl}$ PSS until the responses stabilized. A control contraction was then produced by the addition of $300 \mathrm{nM} \mathrm{NE}$. After sustained tension was obtained, carbachol was added cumulatively $\left(10^{-9}-10^{-5} \mathrm{M}\right)$ to bathing solution in 2-min intervals (relaxation-response curve to carbachol). The Vasodilator effect of increasing concentrations of carbachol was expressed as percent decrease of the peak NE concentration. The $p \mathrm{D}_{2}$ was calculated as the negative logarithm of the dilator concentration that induced $50 \%$ of the maximal relaxation.

\subsection{Statistical Analysis}

All results are expressed as means \pm standard error of mean (SEM) for all groups. In the vasomotor response experiment, the number of rings obtained from different rats is represented by $n$. Relaxation is expressed in terms of the percentage decrease in maximal contraction caused by NE (300 nM). The Prism 5 software (Graph Pad Software, San Diego, CA, USA) was used for the statistical analysis. The differences were analyzed using one-way ANOVA followed by a Dunnett's test. Resulting $P$ values less than 0.05 were regarded as significant. 

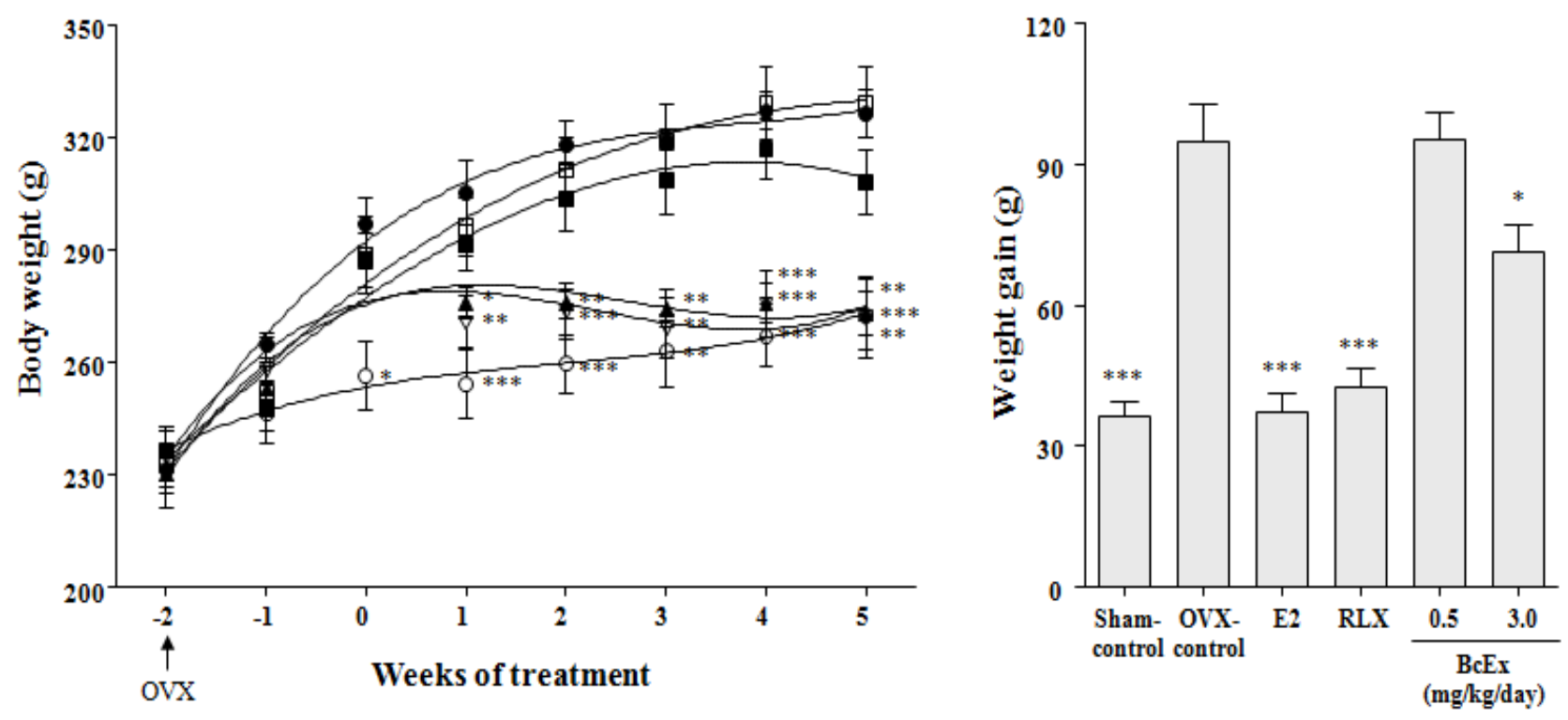

(A) Changes in body weight during experimental periods. Sham-control ( $\circ$, sham-operated group), OVX-control (•, vehicle injected OVX group), E2 $(\nabla, 0.5 \mathrm{mg} / \mathrm{kg}$ /day E2-injected OVX group), RLX $(\boldsymbol{\Lambda}, 2.5 \mathrm{mg} / \mathrm{kg} /$ day RLX-injected OVX group), or BcEx ( $\square$ or $\mathbf{\square}, 0.5 \mathrm{or} 3.0 \mathrm{mg} / \mathrm{kg} / \mathrm{day}$ BcEX-injected OVX group). (B) Body weight gain after 5 weeks of treatments. $* * * P<0.001, * * P<0.01$, and $* P<0.05$ vs. the OVX-control group by one-way ANOVA followed by Dunnett's test ( $n=6-7$, respectively).

Figure 1. Effect of subcutaneously injected BcEx, 17ß-estradiol (E2), and raloxifene (RLX) on body weight in ovariectomized rats

\section{Results}

\subsection{Effects of BcEx, E2, and RLX on Body and Uterine Weights in OVX Rats}

Body weight increased significantly in all OVX groups 2 weeks after surgery, compared with the sham-control group (Figure 1A). The lower concentration $\mathrm{BcEx}$ treatment $(0.5 \mathrm{mg} / \mathrm{kg} /$ day $)$ administered for 5 weeks had an increased effect on body weight gain to the level of the OVX-control group, but injections of E2, RLX, and higher concentrations of $\mathrm{BcEx}(3.0 \mathrm{mg} / \mathrm{kg} /$ day $)$ significantly reduced body weight gain (Figure 1B). The uterine weight of the OVX subgroup decreased significantly in the BcEx and RLX treatment groups compared with the sham-control group, but the E2-treated rats did not differ (Figure 2).

\subsection{Effects of BcEx, E2, and RLX on Serum Lipid Profiles in OVX rats}

The lower concentrations of BcEx in OVX rats did not affect serum total cholesterol (TC), high density lipoprotein-cholesterol (HDL-C), low density lipoproteincholesterol (LDL-C), or triglyceride (TG) levels compared with those in OVX-control rats (Table 1). However, rats that received higher concentrations of $\mathrm{BcEx}$ exhibited significantly lower TC, whereas TG and HDL-C levels remained unaffected. Additionally, rats injected with the higher concentration of BcEx exhibited lower LDL-C levels. RLX also exerted effects on serum TC, LDL-C, and TG levels similar to those of BcEx. In contrast, E2 significantly decreased TC and LDL-C levels, increased TG levels, but did not affect HDL-C levels.

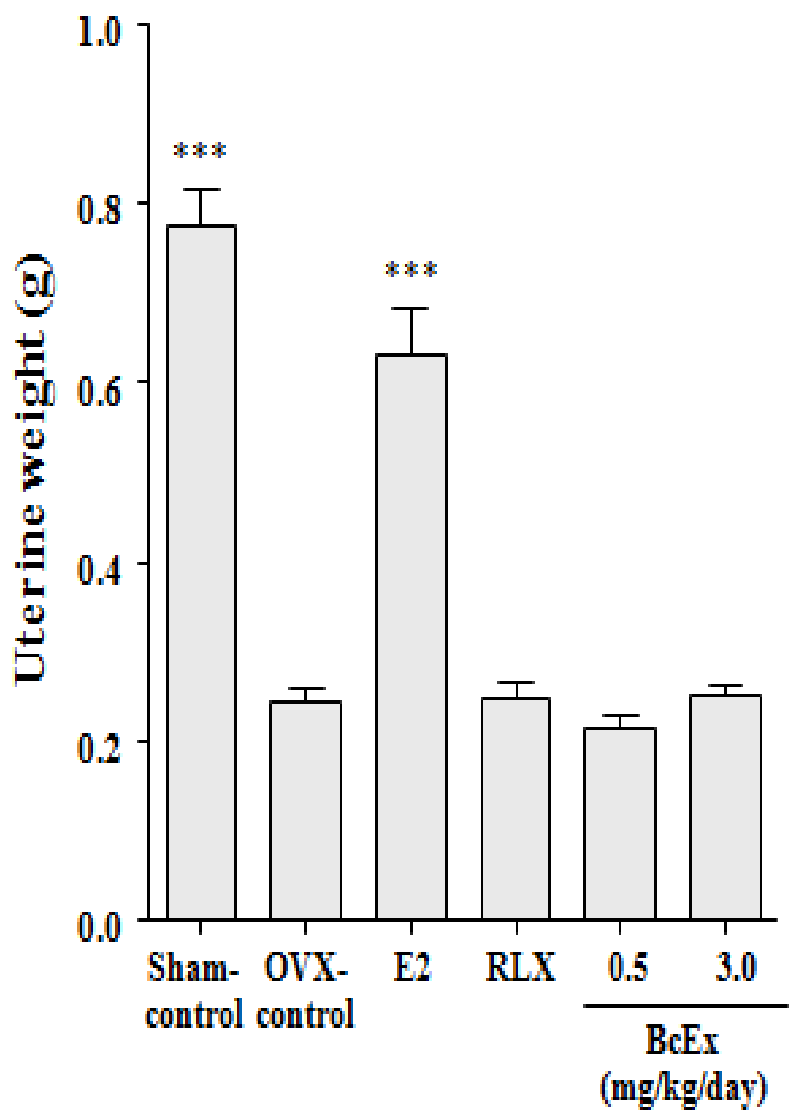

$* * * P<0.001 v s$. the OVX-control group by one-way ANOVA followed by Dunnett's test ( $\mathrm{n}=6-7$, respectively).

Figure 2. Effect of BcEx (0.5 or $3.0 \mathrm{mg} / \mathrm{kg} / \mathrm{day}), 17 \beta$-estradiol (E2, 0.5 $\mathrm{mg} / \mathrm{kg} /$ day), and raloxifene (RLX, $2.5 \mathrm{mg} / \mathrm{kg} /$ day) on uterine weight in ovariectimized rats after 5 weeks of treatments 
Table 1. Effects of subcutaneously injected BcEx (0.5 or $3.0 \mathrm{mg} / \mathrm{kg} / \mathrm{day}), 17 \beta$-estradiol (E2, 0.5 mg/kg/day), and raloxifene (RLX, $2.5 \mathrm{mg} / \mathrm{kg} / \mathrm{day})$ on serum lipid profiles in ovariectomized rats; serum total cholesterol (TC), high-density lipoprotein cholesterol (HDL-C), low-density lipoprotein cholesterol (LDL-C), and triglyceride (TG) levels in sham and OVX rats after 5 weeks of treatments

\begin{tabular}{lccc}
\hline & TC $(\mathrm{mg} / \mathrm{dL})$ & HDL-C $(\mathrm{mg} / \mathrm{dL})$ & LDL-C (mg/dL) \\
\hline Sham-control & $88.14 \pm 8.41^{*}$ & $30.14 \pm 2.71$ & $46.03 \pm 5.45$ \\
OVX-control & $107.20 \pm 5.03$ & $34.00 \pm 2.41$ & $57.92 \pm 1.93$ \\
E2 $(0.5 \mathrm{mg} / \mathrm{kg} /$ day) & $81.60 \pm 3.66^{*}$ & $30.20 \pm 1.39$ & $24.72 \pm 6.11^{* * *}$ \\
RLX (2.5 mg/kg/day) & $78.60 \pm 3.28^{* *}$ & $32.40 \pm 2.32$ & $28.16 \pm 2.48^{* *}$ \\
BcEx (0.5 mg/kg/day) & $107.00 \pm 3.59$ & $32.40 \pm 1.78$ & $59.88 \pm 3.89$ \\
BcEx (3.0 mg/kg/day) & $82.00 \pm 6.57^{*}$ & $26.17 \pm 2.09$ & $90.20 \pm 5.27$ \\
\hline
\end{tabular}

Values are expressed as mean \pm SEM ( $\mathrm{n}=6-7$ per group). Statistical significance is indicated by $* * * P<0.001, * * P<0.01$, and $* P<0.05 v s$. the OVXcontrol group (one-way ANOVA followed by Dunnett's test).

$\mathbf{A}$

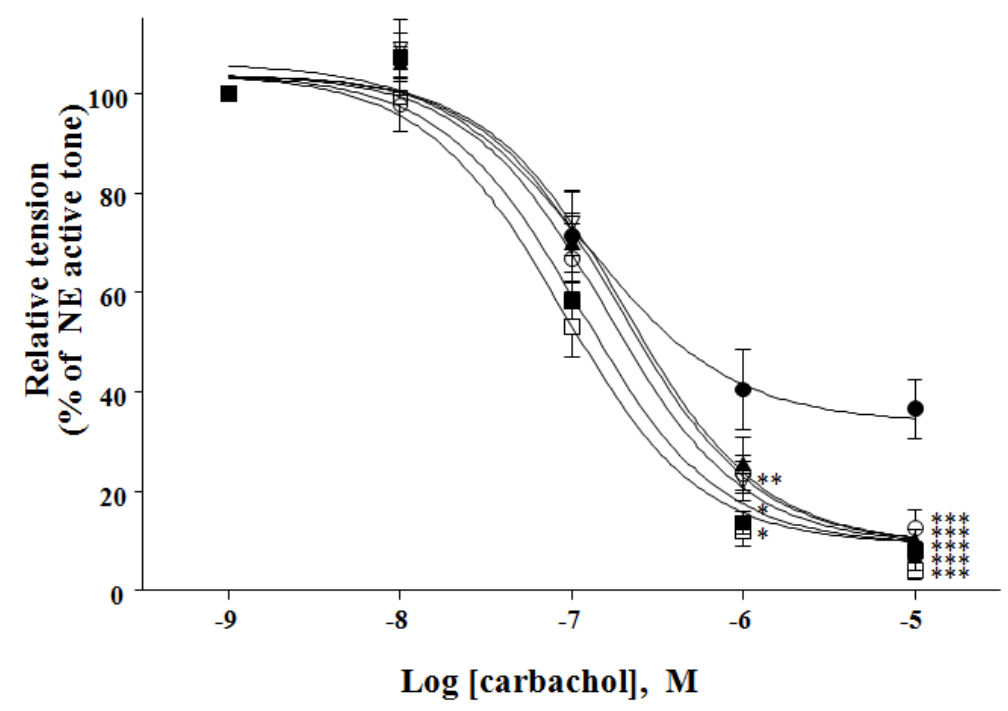

B

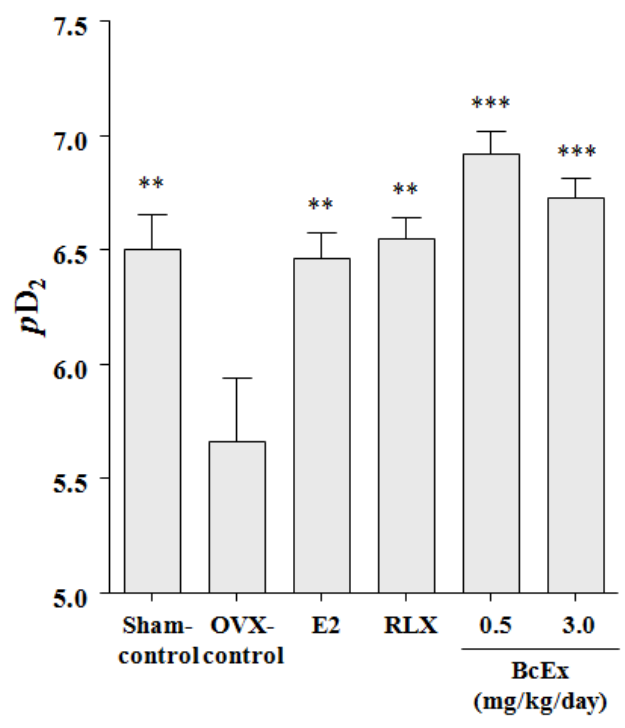

(A) Each symbols indicate the relative tension observed in arteries from sham-operated (Sham-control, $\circ$ ) and ovariectomized rats treated with vehicle

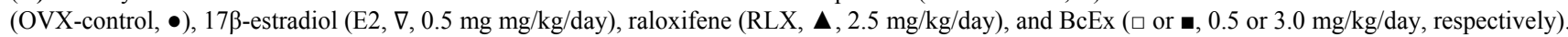
(B) The $p \mathrm{D} 2$ values were calculated as negative logarithm of the dilator concentration that induced $50 \%$ of the maximal relaxation. $* * * P<0.001, * * P<$ 0.01 , and $* P<0.05$ vs. OVX-control group by one-way ANOVA followed by Dunnett's test ( $\mathrm{n}=5-7$, respectively).

Figure 3. Vasomotor responses to carbachol in rat thoracic aorta pre-contracted with $300 \mathrm{nM}$ norepinephrine

\subsection{Effects of BcEx, E2, and RLX on Vasomotor Responses to Carbachol in NE-precontracted Isolated Aortic Rings}

Carbachol relaxed NE-precontracted endothelium-intact rat aortic rings from OVX rats in a dose-dependent manner (Figure 3A). However, the relative magnitude of relaxation in response to carbachol was higher in arteries isolated from rats injected with BcEx, E2, or RLX. The $p \mathrm{D} 2$ value of the relaxation response to carbachol was significantly lower in the OVX-control group compared with that in the other groups (Figure 3B), suggesting that $\mathrm{BcEx}, \mathrm{E} 2$, and RLX improved the vasomotor responses to carbachol in OVX rat aorta.

\section{Discussion}

To our knowledge, this is the first study to compare the effects of BcEx, E2 and RLX on serum lipid profiles and vasomotor responses to those of carbachol in isolated aortic rings contracted by NE. The cardiovascular protective effects of estrogen are mediated by inhibition of body weight gain, atrophy of the uterus and a decrease in serum cholesterol levels [20]. Additionally, estrogen protects against vascular injuries by increasing NO production in damaged arteries [21].

RLX is a second-generation SERM that improves the lipid profiles of menopausal women [22]. Although estrogen exerts cardiovascular protective effects by inhibiting body weight gain [20], the effects of RLX on body weight are a matter of debate [7]. In this study, RLX and E2 treatments attenuated body weight gain, but BcEx did not. Our findings conflict with a previous report that $\mathrm{BcEx}$ attenuated body weight gain in OVX rats [12]. The lower doses of BcEx used in our study $(0.5$ and $3.0 \mathrm{mg} / \mathrm{kg} / \mathrm{day})$, compared with the $135 \mathrm{mg} /$ day used in previous studies, and the difference between the method of administration (subcutaneous injection vs. oral intake) could explain these differences. Estrogen exerts an uterotrophic effect in OVX female rats, but chronic estrogen replacement therapy increases the risk of cancer in reproductive tissues, 
particularly the uterus [23]. However, Seidlova-Wuttke et al. [4] reported that RLX treatment did not increase uterine weight significantly in OVX rats. In this study, neither BcEx nor RLX influenced uterine weight, suggesting that BcEx may act on the OVX rat uterus in a SERM-like manner.

BcEx reduced TC, LDL-C and TG levels in OVX rats in a concentration-dependent manner, albeit to a lesser degree than did RLX. Postmenopausal changes in lipid profiles may contribute to an increased risk of cardiovascular disease [2]. As reported by previous studies, estrogen administration decreased serum TC and LDL-C levels but increased TG levels in OVX rats [24]. TGs are considered to play a key role in cardiovascular risk in postmenopausal women [25]; therefore, increases in TG levels are considered undesirable changes associated with estrogen therapy. In contrast, RLX reduces serum TC and LDL-C levels in humans [26] and TG levels in animal models [7]. Furthermore, clinical studies have reported that RLX reduces LDL-C levels but does not affect TG levels [26]. In this study, BcEx improved serum lipid profiles without increasing TG levels in OVX rats, suggesting that BcEx behaved in a RLX-like manner in terms of its effect on serum lipid profiles.

During the postmenopausal period, decreased NO availability in endothelial cells elicits morphological alterations, which is an important factor in the development of cardiovascular diseases [27]. RLX and E2 exert vasorelaxant effects by upregulating NO production in the endothelium $[6,28]$. In previous studies, we demonstrated that the vasorelaxant effects of BcEx are mediated by enhancement of the NO/cGMP system and inhibition of calcium influx into vascular smooth muscle cells [13]. Additionally, carbachol elicits endothelium-dependent, NO-mediated relaxation in isolated rat aortae [29]. In our study, the vasomotor response to carbachol in isolated aortas contracted by NE was reduced in OVX-control rats compared with sham-control rats. However, BcEx injection significantly increased the $p \mathrm{D} 2$ value of carbachol-induced relaxation to the level of the sham-control, suggesting an improved vasomotor response. Wong et al. [14] showed that OVX did not affect the impaired endothelial function, but that relaxation was transformed to slight contraction in response to higher concentrations of acetylcholine. Additionally, Lamas et al. [30] suggested that RLX and E2 treatments restored the decreased acetylcholine-induced relaxant responses in OVX rats. Therefore, BcEx improved vasomotor responses in OVX rats in a manner similar to that by RLX and E2.

\section{Conclusion}

The findings of this study demonstrate that $\mathrm{BcEx}$ improved serum lipid profiles and increased the vasomotor responses to carbachol in aortic rings from OVX rats. Although the effects of BcEx on serum lipid profiles were similar to those of RLX rather than those of E2, the vasomotor responses to $\mathrm{BcEx}$ were consistent with those to E2 and RLX. Therefore, our results suggest that longterm administration of $\mathrm{BcEx}$ exerts cardioprotective effects by improving serum lipid profiles and vasomotor responses via a SERM-like mechanism.

\section{Acknowledgments}

This study was supported by Korea Institute of Planning \& Evaluation for Technology in Food, Agriculture, Forestry \& Fisheries, Republic of Korea (GA0957) and National Research Foundation of Korea (NRF) grant NRF-2017R1A2B2008527.

\section{Conflicts of Interest}

The authors declare no conflicts of interest.

\section{References}

[1] Palacios, S, "Current perspectives on the benefits of HRT in menopausal woman," Maturitas, 33 (Suppl 1). S1-S13. Nov 1999.

[2] Stampfer, M.J., Colditz, G.A., Willett, W.C., Manson, J.E., Rosner, B. and Speizer, F.E, "Postmenopausal estrogen therapy and cardiovascular disease: Ten-year follow-up from the nurses' health study", New England Journal of Medicine, 325 (11). 756-762. Sep 1991.

[3] Wuttke, W., Jarry, H., Becker, T., Schultens, A., Christoffel, V., Gorkow, C. and Seidlova-Wuttke, D, "Phytoestrogens: endocrine disrupters or replacement of hormone replacement therapy?", Maturitas, 44 (Suppl 1). S9-S20. Mar 2003.

[4] Seidlova-Wuttke, D., Jarry, H. and Wuttke, W, "Effects of estradiol benzoate, raloxifene and an ethanolic extract of Cimicifuga racemosa in nonclassical estrogen regulated organs of ovariectomized rats", Planta Medica, 75 (12). 1279-1285. Oct 2009.

[5] Anthony, M., Williams, J.K. and Dunn, B.K, "What would be the properties of an ideal SERM?", Annals New York Academy of Sciences, 949. 261-278. Dec 2001.

[6] Bracamonte, M.P., Rud, K.S. and Miller, V.M, "Mechanism of raloxifene-induced relaxation in femoral veins depends on ovarian hormonal status", Journal of Cardiovascular Pharmacology, 39 (5). 704-713. May 2002

[7] Sutherland, M.K., Brady, H., Gayo-Fung, L.M., Leisten, J., Lipps, S.G., McKie, J.A., O'Leary, E., Patnaik, N., Anderson, D.W., Bhagwat, S.S. and Stein, B, "Effects of SP500263, a novel selective estrogen receptor modulator, on bone, uterus, and serum cholesterol in the ovariectomized rat", Calcified Tissue International, 72 (6). 710-716. Jun 2003.

[8] Borrelli, F. and Ernst, E, "Cimicifuga racemosa: a systematic review of its clinical efficacy", European Journal of Clinical Pharmacology, 58 (4). 235-241. Jul 2002.

[9] Osmers, R., Friede, M., Liske, E., Schnitker, J., Freudenstein, J. and Henneicke-von Zepelin, H.H, "Efficacy and safety of isopropanolic black cohosh extract for climacteric symptoms", Obstetrics and Gynecology, 105 (5 Pt 1). 1074-1083. May 2005.

[10] Geller, S.E., Shulman, L.P., Breemen, R.B., Banuvar, S., Zhou, Y., Epstein, G., Hedayat, S., Nikolic, D., Krause, E.C., Piersen, C.E., Bolton, J.L., Pauli, G.J. and Farnsworth, N.R, "Safety and efficacy of black cohosh and red clover for the management of vasomotor symptoms: a randomized controlled trial", Menopause, 16 (6). 1156-1166. Nov-Dec 2009.

[11] Seidlova-Wuttke, D., Jarry, H., Becker, T., Christoffel, V. and Wuttke, W, "Pharmacology of Cimicifuga racemosa extract BNO 1055 in rats: bone, fat and uterus", Maturitas, 44 (Suppl 1). S39-S50. Mar 2003.

[12] Rachon, D., Rortherms, T., Seidlova-Wuttke, D. and Wuttke, W, "Effects of black cohosh extract on body weight gain, intra-abdominal fat accumulation, plasma lipids and glucose tolerance in ovariectomized Sprageu-Dawley rat", Maturitas, 60 (3-4). 209-215. Jul-Aug 2008.

[13] Kim, E.Y., Lee, Y.J. and Rhyu, M.R, "Black cohosh (Cimicifuga ragemosa) relaxes the isolated rat thoracic aorta through endothelium-dependent and -independent mechanisms", Journal of Ethnopharmacology, 138 (2). 537-542. Nov 2011.

[14] Wong, C.M., Yao, X., Au, C.L., Tsang, S.Y., Fung, K.P., Laher, I, Vanhoutte, P.M. and Huang, Y, "Raloxifene prevents endothelial dysfunction in aging ovariectomized female rats", Vascular Pharmacology, 44 (5). 290-298. Mar 2006. 
[15] Simoncini, T., Genazzani, A.R. and Liao, J.K, "Nongenomic mechanisms of endothelial nitric oxide synthase activation by the selective estrogen receptor modulator raloxifene", Circulation, 105 (11). 1368-1373. Mar 2002.

[16] Rhyu, M.R., Lu, J., Webster, D.E., Fabricant, D.S., Farnsworth N.R. and Wang, Z.J, "Black cohosh (Actaea racemosa, Cimicifuga racemosa) behaves as a mixed competitive ligand and partial agonist at the human $\mu$ opiate receptor", Journal of Agricultural and Food Chemistry, 54 (26). 9852-9857. Dec 2006.

[17] Burdette, J.E., Liu, J., Chen, S.N., Fabricant, D.S., Piersen, C.E., Barker, E.L., Pezzuto, J.M., Mesecar, A., Breemen, R.B., Farnsworth, N.R. and Bolton, J.L, "Black cohosh acts as a mixed competitive ligand and partial agonist of the serotonin receptor", Journal of Agricultural and Food Chemistry, 51 (19). 5661-5670. Sep 2003.

[18] Xu, H., Fabricant, D.S., Piersen, C.E., Bolton, J.L., Mezzute, J.M., Fong, H., Totura, S., Farnsworth, N.R. and Constantinou, A.I, "A preliminary RAPD-PCR analysis of Cimicifuga species and other botanicals used for women's health", Phytomedicine, 9 (8). 757-762. Dec 2002.

[19] Friedwald, W., Levy, R. and Fredrickson, D, "Estimation of the concentration of low density lipoprotein in plasma without use of preparative ultracentrifuge", Clinical Chemistry, 18 (6). 499-502. Jun 1972.

[20] Frolik, C.A., Bryant, H.U., Black, E.C., Magee, D.E. and Chandrasekhar, S, "Time-dependent changes in biochemical bone markers and serum cholesterol in ovariectomized rats: effects of raloxifene $\mathrm{HCl}$, tamoxifen, estrogen, and alendronate", Bone, 18 (6). 621-627. Jun 1996.

[21] White, C.R., Shelton, J., Chen, S.J., Darley-Usmar, V., Allen, L., Nabors, C., Sanders, P.W., Chen, F. and Oparil, S, "Estrogen restores endothelial cell function in an experimental model of vascular injury", Circulation, 96 (5). 1624-1630. Sep 1997.

[22] Delmas, P.D., Bjarnason, N.H., Mitlak, B.H., Ravoux, A.C., Shah, A.S., Huster, W.J., Draper, M. and Christiansen, C, "Effects of raloxifene on bone mineral density, serum cholesterol concentrations, and uterine endometrium in postmenopausal women", New England Journal of Medicine, 337 (23). 1641-1647. Dec 1997.

[23] Hammond, C.B, "Women's concerns with hormone replacement therapy: Compliance issues", Fertility and Sterility, 62 (6 Suppl 2). 157S-160S. Dec 1994.

[24] Ateba. S.B., Njamen, D., Medjakovic, S., Hobiger, S., Mbanya, J.C., Jungbauer, A. and Krenn, L, "Eriosema laurentii De Wild (leguminosae) methanol extract has estrogenic properties and prevents menopausal symptoms in ovariectomized Wistar rats", Journal of Ethnopharmacology, 150 (1). 298-307. Oct 2013.

[25] Christodoulakos, G.E., Lambrinoudaki, I.V. and Botsis, D.C, "The cardiovascular effects of selective estrogen receptor modulators", Annals New York Academy of Science, 1092. 374-384. Dec 2006.

[26] Walsh, B.W., Kuller, L.H., Wild, R.A., Paul, S., Farmer, M., Lawrence, J.B., Shah, A.S. and Anderson, P.W, "Effects of raloxifenen on serum lipids and coagulation factors in healthy postmenopausal women" JAMA, 279 (18). 1445-1451. May 1998.

[27] Arenas, L.A., Armstrong, S.J., Xu, Y. and Davidge, S.T, "Chronic tumor necrosis is factor-alpha inhibition enhances NO modulation of vascular function in estrogen-deficient rats", Hypertension, 46 (1). 76-81. Jul 2005.

[28] Figtree, G.A., Lu, Y., Webb, C.M. and Collins, P, "Raloxifene acutely relaxes rabbit coronary arteries in vitro by an estrogen receptor-dependent and nitric oxide-dependent mechanism" Circulation, 100 (10). 1095-1101. Sep 1999.

[29] Paredes-Carbajal, M.C., Juarez-Oropeza, M.A., Ortiz-Mendoza, C.M. and Mascher, D, "Effects of acute and chronic estrogenic treatment on vasomotor responses of aortic rings from ovariectomized rats", Life Sciences, 57 (5). 473-486. Jun 1995.

[30] Lamas, A.Z., Caliman, I.F., Dalpiaz, P.L.M., Ferreira de Melo, Jr. A., Abreu, G.R., Lemos, E.M., Gouvea, S.A. and Bissoli, N.S, "Comparative effects of estrogen, raloxifene and tamoxifen on endothelial dysfunction, inflammatory markers and oxidative stress in ovariectomized rats”, Life Sciences, 124 (1). 101-109. Mar 2015. 